\title{
The high frequency of GJB2 gene mutation c.313_326del14 suggests its possible origin in ancestors of Lithuanian population
}

Violeta Mikstiene ${ }^{1 *} \mathbb{D}$, Audrone Jakaitiene ${ }^{1}$, Jekaterina Byckova², Egle Gradauskiene ${ }^{2}$, Egle Preiksaitiene ${ }^{1}$, Birute Burnyte ${ }^{1}$, Birute Tumiene ${ }^{1}$, Ausra Matuleviciene ${ }^{1}$, Laima Ambrozaityte ${ }^{1}$, Ingrida Uktveryte ${ }^{1}$, Ingrida Domarkiene ${ }^{1}$, Tautvydas Rancelis ${ }^{1}$, Loreta Cimbalistiene ${ }^{1}$, Eugenijus Lesinskas ${ }^{2}$, Vaidutis Kucinskas ${ }^{1}$ and Algirdas Utkus ${ }^{1}$

\begin{abstract}
Background: Congenital hearing loss $(\mathrm{CHL})$ is diagnosed in $1-2$ newborns in 1000, genetic factors contribute to two thirds of CHL cases in industrialised countries. Mutations of the GJB2 gene located in the DFNB1 locus (13q11-12) are a major cause of $\mathrm{CHL}$ worldwide.

The aim of this cross-sectional study was to assess the contribution of the DFNB1 locus containing the GJB2 and GJB6 genes in the development of early onset hearing loss in the affected group of participants, to determine the population-specific mutational profile and DFNB1-related HL burden in Lithuanian population.

Methods: Clinical data were obtained from a collection of 158 affected participants (146 unrelated probands) with early onset non-syndromic HL. GJB2 and GJB6 gene sequencing and GJB6 gene deletion testing were performed. The data of GJB2 and GJB6 gene sequencing in 98 participants in group of self-reported healthy Lithuanian inhabitants were analysed. Statistic summary, homogeneity tests, and logistic regression analysis were used for the assessment of genotype-phenotype correlation.

Results: Our findings show $57.5 \%$ of affected participants with two pathogenic GJB2 gene mutations identified. The most prevalent GJB2 mutations were c.35delG, p. (Gly12Valfs*2) (rs80338939) and c.313_326del14, p. (Lys105Glyfs*5) (rs111033253) with allele frequencies $64.7 \%$ and $28.3 \%$ respectively. GJB6 gene mutations were not identified in the affected group of participants. The statistical analysis revealed significant differences between GJB2(-) and GJB2(+) groups in disease severity $(p=0.001)$, and family history $(p=0.01)$. The probability of identification of GJB2 mutations in patients with various $\mathrm{HL}$ characteristics was estimated. The carrier rate of GJB2 gene mutations $-7.1 \%(\sim 1$ in 14) was identified in the group of healthy participants and a high frequency of GJB2-related hearing loss was estimated in our population.

(Continued on next page)
\end{abstract}

\footnotetext{
* Correspondence: violeta.mikstiene@mf.vu.lt

${ }^{1}$ Department of Human and Medical Genetics, Faculty of Medicine, Vilnius

University, Vilnius, Lithuania

Full list of author information is available at the end of the article
} 
(Continued from previous page)

Discussion: The results show a very high proportion of GJB2-positive individuals in the research group affected with sensorineural HL. The allele frequency of c.35delG mutation (64.7\%) is consistent with many previously published studies in groups of affected individuals of Caucasian populations. The high frequency of the c.313_326del14 (28.3\% of pathogenic alleles) mutation in affected group of participants was an unexpected finding in our study suggesting not only a high frequency of carriers of this mutation in our population but also its possible origin in Lithuanian ancestors. The high frequency of carriers of the c.313_326del14 mutation in the entire Lithuanian population is supported by it being identified twice in the ethnic Lithuanian group of healthy participants (a frequency $2.0 \%$ of carriers in the study group).

Conclusion: Analysis of the allele frequency of GJB2 gene mutations revealed a high proportion of c. 313_326del14 (rs111033253) mutations in the GJB2-positive group suggesting its possible origin in Lithuanian forebears. The high frequency of carriers of GJB2 gene mutations in the group of healthy participants corresponds to the substantial frequency of GJB2-associated HL in Lithuania. The observations of the study indicate the significant contribution of GJB2 gene mutations to the pathogenesis of the disorder in the Lithuanian population and will contribute to introducing principles to predict the characteristics of the disease in patients.

Keywords: Non-syndromic sensorineural hearing loss, GJB2 and GJB6 genes, c.313_326del14 mutation, p.(Lys105Glyfs*5), Frequency of carriers of GJB2 gene mutation in the Lithuanian population

\section{Background}

Congenital hearing loss (CHL) is a disease of considerable concern in medicine nowadays. It is one of the most common conditions and is diagnosed in $1-2$ of 1000 newborns [1]. The incidence rises to 3.5 of 1000 before adolescence [2]. The disorder is highly heterogeneous; every population has a unique HL etiologic profile dependent on ethnic, geographic, social and medical factors. Genetic factors contribute to up to two thirds of CHL cases in industrialized countries [3]. Most cases, about $70 \%$, have non-syndromic hearing loss and about $30 \%$ represent syndromic deafness [4]. The remaining one-third of cases can be ascribed to environmental and unidentified genetic factors.

At least 400 and over 150 genetic loci are associated with syndromic and non-syndromic hearing loss respectively $[5,6]$. The inheritance of the disorder may be autosomal dominant, autosomal recessive, $\mathrm{X}$ recessive and mitochondrial.

Pathogenic mutations in the DFNB1 (Deafness) locus (13q11-12) containing GJB2 (NM_004004.5) and GJB6 (NM_001110219.2) genes are the most common cause of non-syndromic sensorineural hereditary hearing loss worldwide [7]. Results of the analysis of the DFNB1 locus in patients in different populations demonstrate the leading role of that pathogenic changes in the GJB2 and GJB6 genes, which account from 10 to $40 \%$ of cases, have in etiologic profile of sensorineural HL [8].

To date more than 300 pathogenic mutations of the GJB2 gene and over 20 pathogenic mutations including gross del(GJB6-D13S1830) and $\operatorname{del}(G J B 6-D 13 S 1854)$ in the GJB6 gene have been determined leading to development of sensorineural HL [9].
GJB2 and GJB6 genes undergo coordinated transcription, and their major expressing organs are cochlea, placenta, hepatocytes, skin, pancreas, kidney and intestine (GJB2 gene), and astrocytes, cochlea (GJB6 gene) [10]. Connexin 26, a 226 amino acid protein encoded by the GJB2 gene (OMIM* 121011) and connexin 30, 261 amino acid protein encoded by the GJB6 gene (OMIM* 604418), form connexons. These homo- or heteromeric structures compose gap junctions and are essential in the transport of $\mathrm{K}^{+}, \mathrm{Ca}^{2+}$ ions, $\mathrm{IP}_{3}$, and other small molecules between many cells in an organism including the supporting cells in the inner ear, and provide a direct pathway of communication for intercellular electrical and chemical signaling. The altered recycling of $\mathrm{K}^{+}$to the endolymph of the cochlea disturbs the repolarization of the hair cell membrane and formation of an auditory nerve impulse [11].

The disease characteristics (severity, symmetry, age of onset, etc.) of sensorineural HL linked with DFNB1 vary and were shown to be dependent on the GJB2 and/or GJB6 genotype and the nature of the pathogenic mutation [12]. Most GJB2 and GJB6 mutations are associated with autosomal recessive non-syndromic hearing loss, but several dominant mutations, causing sensorineural HL or syndromic hearing loss (KID (MIM 148210), Vohwinkel (MIM 124500) syndromes, palmoplantar keratoderma (MIM 148350)) have been described [13].

Analysis of the mutational spectrum in the DFNB1 locus, genotype - phenotype correlation, and analysis of the burden of DFNB1-related hearing loss have been performed in many countries. The aim of our crosssectional study was to assess the contribution of GJB2 and GJB6 gene mutations to the development of early onset hearing loss and determine the mutational profile 
in the affected group of participants in the Lithuanian population and to analyse the burden of GJB2 and GJB6 gene mutations in our population, adding missing puzzle piece to the genetics of congenital hearing loss.

\section{Methods}

\section{Study design}

We performed cross-sectional study using data of the two observational projects: DEAFGEN and LITGEN. One of the aims of DEAFGEN is to identify pathogenic mutations of known genes associated with hereditary hearing loss and characterize their phenotypes. The aim of the LITGEN project was to perform wide scale genomic studies of the population of Lithuania and to identify genomic regions of hypothetical Lithuanian which are significant for health. Data and results of whole genome, whole exome and genome-wide genotyping becomes the background for the reference genome of the population of Lithuania and a variety of studies related with monogenic and complex diseases in the population of Lithuania.

We enrolled two groups of participants (DEAFGEN group: individuals affected with early onset HL and LITGEN group: individuals of ethnic Lithuanian population) in the current study, and collected data and venous blood samples for the clinical and genetic analysis. Population-specific DFNB1 locus mutation profile was determined in both groups of participants, genotype - phenotype correlation analysis performed in affected group of participants (DEAFGEN), and burden of DFNB1-related HL in our population assessed (LITGEN).

\section{Recruitment of participants with non-syndromic sensorineural HL (DEAFGEN project)}

Patients affected with early onset (before 5 years of age) non-syndromic hearing loss referred to the Vilnius University Hospital Santariskiu Clinics Centre for Medical Genetics and Centre of Ear, Nose and Throat from 2010 to 2015 were enrolled in this study.

Demographic data and medical records were obtained and physical examination and genealogy analysis were performed. In the presence of several affected relatives in the family, only one (randomly chosen) was recruited to the study to avoid bias of analysis.

Subgroups of affected participants were formed according to the results of the analysis of disease severity, symmetry and three generation genealogy. The participant was assigned to the positive genealogy subgroup if at least one relative with early onset hearing loss was determined in the family or negative genealogy subgroup - if the case was apparently sporadic.

\section{Clinical evaluation of the affected group}

All participants in the study were assessed in accordance with age-specific specialised audiological evaluations.
Pure-tone audiometry was obtained when possible, with the use of a diagnostic audiometer in a soundproof booth, in accordance with ISO standards. The threshold values in decibels $(\mathrm{dB})$ for $0.5,1,2$ and $4 \mathrm{kHz}$ were averaged for both ears (pure-tone average PTA). In cases without pure-tone audiometry, the threshold of the wave $\mathrm{V}$ of the click-evoked auditory brainstem responses $A B R$ or auditory steady state response ASSR were used to calculate the hearing level. The definition of the degree and type of $\mathrm{HI}$ was based on the most recent audiogram available. The degree of HL was classified according to the PTA (or extrapolated auditory brainstem responses value) as mild (21-40 dB), moderate (41-70 dB), severe (71-90 dB), or profound ( $>90 \mathrm{~dB})$. The severity of deafness was defined by the degree of hearing loss in the better ear. Asymmetry was defined if the PTA between ears revealed the difference of $15 \mathrm{~dB}$ or greater.

Venous blood samples and written informed consent forms of affected participants or their parents (in the case of minors under the age of 16 years) were collected for the 'The genomics of congenital/hereditary hearing loss: implication in disease pathogenesis, influence to phenotypic expression and treatment efficiency' (acronym: DEAFGEN) project. The approval to conduct the DEAFGEN project was provided by the Vilnius Regional Research Ethics Committee.

\section{Recruitment of ethnic Lithuanian population group of healthy individuals (LITGEN project)}

The group of healthy participants consisted of 98 unrelated adult individuals. This group represents the pure ethnic Lithuanian population due to the strict criteria of the enrolment conferring the uniqueness of this group: all self-reported healthy study participants indicated at least three generations of Lithuanian ethnicity and residency in the same ethno-linguistic region.

The data, venous blood samples and written informed consent forms were collected from individuals (trios) who were invited to the primary healthcare centers in the different regions of Lithuania (West, North, South Zemaitija and West, East, South Aukstaitija) in the period 2011-2013 for the 'Genetic diversity of the population of Lithuania and changes of its genetic structure related with evolution and common diseases' (acronym: LITGEN) project. The approval to conduct the LITGEN project was provided by the Vilnius Regional Research Ethics Committee. No follow-up or exposure was performed.

\section{Genetic analysis in affected group of participants}

Whole genomic DNA was extracted from peripheral blood following the standard phenol-chloroform extraction protocol. 
Polymerase chain reactions (PCR) of coding sequence and sequences flanking splicing site mutation c. $-23+1 G>A$ (rs80338940) of the GJB2 and GJB6 genes were performed using specific primers designed with Primer Blast (NCBI) software [14] (see Additional file 1). Both strands of PCR products were sequenced with BigDye ${ }^{\bullet}$ Terminator v3.1 Cycle Sequencing Kit (Applied Biosystems, USA). Capillary electrophoresis was carried out with 3130xL Genetic Analyser (Applied Biosystems, USA). Fluorescent signals were analysed with Sequence Analysis v5.1 software (Applied Biosystems, USA). The sequences obtained were aligned with the reference sequence of the GJB2 (NCBI NM_004004.5) gene. The sequence variants were analyzed in the Human Gene Mutation Database [15] and Connexin Deafness Homepage [16] Segregation analysis was performed by sequencing the GJB2 gene to the parents of the affected participants.

The multiplex PCR assay designed by del Castillo [17] was used to detect the del(GJB6-D13S1830) and del(GJB6D13S1854) mutations in the group of affected participants if GJB2 mutations were not identified or only one heterozygous GJB2 mutation was identified.

The frequencies of the DFNB1 mutations were defined and inactivating as well as non-inactivating mutations assessed. After the genetic testing, three major subgroups GJB2(+), GJB2(+/-) and GJB2(-) were formed to perform the statistical analysis of genotype - phenotype correlation. GJB2(+) subgroup was divided into classes of genotypes according to possession of inactivating (frameshift) or non-inactivating (missense) mutation to determine their different impact on the characteristics of the disease.

Genetic analysis in group of ethnic Lithuanian population Genomic DNA was extracted from blood using the phenol-chloroform extraction method or automated nucleic acid purification using paramagnetic particles (TECAN Freedom EVO ${ }^{\circ}$ 200, Tecan Schweiz AG, Switzerland). Next-generation exome sequencing after insolution capture enrichment (TargetSeq ${ }^{\mathrm{Tw}}$, Life Technologies or SureSelect, Agilent) with an average of a 40-fold coverage was performed at the Department of Human and Medical Genetics, Faculty of Medicine, Vilnius University with the use of a 5500 SOLiD $^{\text {tw }}$ Sequencer according to the optimised manufacturer's protocols. Sequence alignment and secondary and tertiary analysis performed using LifeScope ${ }^{\mathrm{Tm}}$ Genomic Analysis Software v2.5. The Genome Analysis Toolkit's (GATK) CombineVariants tool $[18,19]$ was used to combine all identified genomic variants from 98 individuals into single VCF file. The genomic variants identified were annotated using the Annovar v.2015mar22 [20] program. Each identified GJB2 and GJB6 gene variant was checked by analysing individuals' BAM files using the visualisation tool Integrative Genomics Viewer (IGV) [21].

\section{Statistical analysis}

Hearing loss characteristics (severity and symmetry), family history and allele frequencies were treated as outcome variables in the analysis. Results of genetic testing (GJB2 and GJB6 genotypes) were treated as predictors.

Categorical variables were expressed as absolute numbers and percentages. The binomial exact test was applied to calculate a confidence interval $95 \%$ for a proportion. The homogeneity hypothesis between two variables was tested using Pearson's chi-square. Logistic regression analysis was conducted to assess the impact of GJB2 gene mutations on HL severity and positive family history. P-values less than 0.05 were considered statistically significant. The statistical software package $\mathrm{R}$ (version 3.2.1) was used to obtain the results. G*Power (version 3.1) was used for post hoc power analysis of the test employed.

\section{Results}

Affected group of participants

One hundred fifty-eight participants (146 unrelated probands), 77 female and 81 male with non-syndromic early onset (all children aged under five) hearing loss were enrolled in the affected group of the research. The anonymized data of GJB2 gene genotypes are provided in the Dataset of the group of affected individuals (see Additional file 2, the number given to each participant does not enable his identification).

\section{Results of clinical evaluation in affected group of participants}

According to clinical evaluation profound, severe, moderate and mild hearing loss was identified in 85 (53.8\%), $24(15.2 \%), 37(23.4 \%)$, and $12(7.6 \%)$ of the affected participants respectively.

One hundred forty $(88.6 \%)$ individuals suffered from symmetrical hearing loss and 18 (11.4\%) had nonsymmetric HL.

Genealogy analysis revealed 61 (41.8\%) unrelated participants with positive family history of early onset hearing loss and 85 (58.2 \%) individuals without affected family members (Table 1 ).

\section{Research group of ethnic Lithuanian population}

The group of the ethnic Lithuanian population consisted of 98 unrelated, self-reported healthy individuals (49 female and 49 male participants). The anonymised data with GJB2 and GJB6 genes genotypes are provided in the Dataset of the group of ethnic Lithuanian population (see Additional file 3, 
Table 1 The results of clinical evaluation

\begin{tabular}{cllr}
\hline Feature & Type & Counts & $\%$ \\
\hline Severity & Mild & 12 & 7.6 \\
& Moderate & 37 & 23.4 \\
& Severe & 24 & 15.2 \\
Symmetry & Profound & 85 & 53.8 \\
& Symmetric & 140 & 88.6 \\
Genealogy & Non-symmetric & 18 & 11.4 \\
& Positive & 61 & 41.8 \\
& Negative & 85 & 58.2 \\
\hline
\end{tabular}

the number given to each participant does not enable his identification).

\section{Power analysis}

\section{Affected group of participants}

The post hoc power analysis was performed for all the tests in the study. Calculated empirical effect size ranged from medium to large. The empirical power of the tests was above 0.8. We present values for the empirical effect size and power in corresponding Tables.

\section{Ethnic Lithuanian group}

Assuming medium effect size for the binomial exact test and having sample size of 98, the calculated power is above 0.8 .

\section{GJB2 gene mutation spectrum in affected group of participants}

GJB2 gene coding sequence analysis revealed 2 pathogenic mutations in homozygous or compound heterozygous state in $84(57.5 \%)$ affected unrelated participants, $5(3.4 \%)$ individuals had 1 mutation in heterozygous state and 57 (39.1\%) unrelated participants had no causative GJB2 gene mutations (Fig. 1).

A total of seven different pathogenic mutations (frameshift and missense) were identified in the affected group of participants (Table 2).

The most prevalent GJB2 gene mutation in our study group was c.35delG, p.(Gly12Valfs*2) (rs80338939), which accounts for $64.7 \%$ of pathogenic alleles. This change was identified in a homozygous state in 37 unrelated affected individuals (44.0\% of the GJB2-positive group), also in a heterozygous state with c.313_326del14, p.(Lys105Glyfs*5) (rs111033253) in 28 participants (33.3\% of the GJB2-positive group) and with other mutations in 8 participants $(9.5 \%$ in GJB2-positive group) (Table 3 ).

The second most frequent mutation in the group of affected participants was c.313_326del14, p.(Lys105Glyfs*5) (rs111033253) with a frequency $28.3 \%$ of pathogenic alleles. Nine unrelated affected individuals $(10.7 \%$ of the

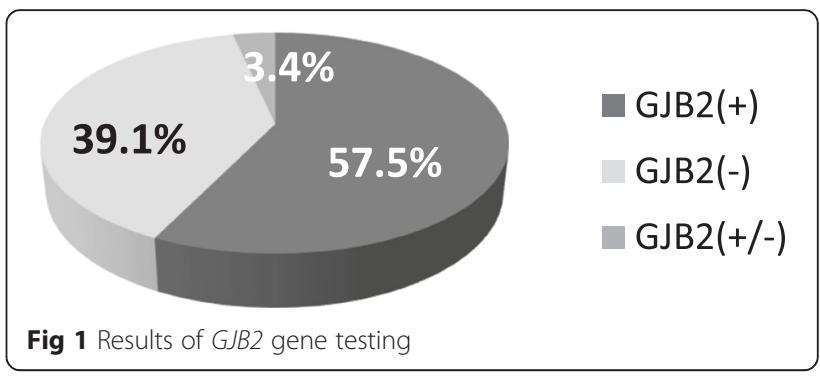

GJB2-positive group) possess the mutation in homozygous state, 28 individuals (33.3\%) - possess it in a heterozygous state with c.35delG, p.(Gly12Valfs*2) (rs80338939) and 2 participants possess it in compound with other mutation $(2.4 \%)$.

Other pathogenic mutations: c.269 T > C, p.(Leu90Pro) (rs8033894), c.101 T C, p.(Met34Thr) (rs35887622), and c.109G > A, p.(Val37Ile) (rs72474224) were much rarer - each accounted for only up to $2.3 \%$ of pathogenic alleles and were in a heterozygous state with either of the two most frequent mutations. The variant of unknown significance, c.379C > T, p.Arg127Cys (rs727503066), was assigned to pathogenic mutations due to a previous publication [22] and our observations (see Discussion).

\section{GJB2 gene mutation spectrum in ethnic Lithuanian population group}

Data of GJB2 gene coding exon sequencing of 98 unrelated participants were analysed.

The results revealed the heterozygous state of GJB2 mutations in 7 DNA samples (7.14 \%) showing that approximately 1 in 14 individuals in the Lithuanian population is a carrier of the GJB2 gene mutation (Table 4). Three healthy study participants had the c.101 T>C, p.(Met34Thr) (rs35887622) mutation (3.1\%), and the mutations c.313_326del14, p.(Lys105Glyfs*5) (rs111033253) and c.35delG, p.(Gly12Valfs*2) (rs80338939) were discovered in a heterozygous state with $2.0 \%$ and $1.0 \%$ carrier frequencies respectively. A novel, previously undescribed truncating mutation c.206delT (p.Phe69Serfs*13) was identified in one participant (genotype frequency $1.0 \%$ ).

According to the frequency of carriers of the GJB2 gene mutation in healthy group of our study $(7.1 \%$ or $\sim 1$ in 14$)$ we estimated the rate of GJB2-related sensorineural HL in Lithuanian population - at approximately 1 in 772 in case of non-assortative marriages.

\section{Results of testing GJB6 gene point mutations and gross deletions}

GJB6 gene point pathogenic mutations and deletions $\operatorname{del}(G J B 6-D 13 S 1830)$ and $\operatorname{del}(G J B 6-D 13 S 1854)$ were not identified in our affected group of participants.

The GJB6 gene mutation c.428G > A, p.(Arg143Gln) (rs201783640) was identified in a heterozygous state in 
Table 2 Allele frequencies of pathogenic GJB2 gene mutations in the affected group of unrelated participants

\begin{tabular}{llll}
\hline Pathogenic GJB2 gene mutation & Count & Allele frequency (\%) & Prediction by in silico computational analysis \\
\hline c.35delG, p.(Gly12Valfs*2), rs80338939 & 112 & 64.7 & MutationTaster2 Disease causing \\
c.313_326del14 p.(Lys105Glyfs*5), rs111033253 & 49 & 28.3 & MutationTaster2 Disease causing \\
c.269 T > C p.(Leu90Pro), rs8033894 & 4 & 2.3 & Sift Damaging (score 0.000) \\
& & & Polyphen-2 Probably damaging (score 1.0) \\
& 4 & 2.3 & MutationTaster2 Disease causing \\
c.101 T > C p.(Met34Thr), rs35887622 & & Sift Damaging (score 0.027) \\
& 2 & & Polyphen-2 Benign (score 0.083) \\
c.167delT p.(Leu56Argfs*26), rs80338942 & 1 & 1.2 & MutationTaster2 Disease causing \\
c.109G > A p.(Val37lle), rs72474224 & 0.6 & MutationTaster2 Disease causing \\
& & & Sift Tolerated (score 0.717) \\
c.379C > T p.(Arg127Cys), rs727503066 & 1 & Polyphen-2 Probably damaging (score 1.0) \\
& & 0.6 & MutationTaster2 Disease causing \\
Total & & & Sift Damaging (score 0.0) \\
\hline
\end{tabular}

one DNA sample in the study group of healthy individuals (carrier frequency $\sim 1.0 \%$ ). The change was evaluated by in silico analysis: SIFT predicted that amino acid substitution likely affects protein function with score 0.003, Polyphen2 predicted probably damaging with score 0.998[23]; MutationTaster2 predicted the change to be disease causing.

\section{Genotype - phenotype correlation analysis}

To analyse genotype - phenotype correlation the group of affected participants was divided into three major subgroups according to the results of GJB2 gene sequencing. The GJB2-positive subgroup consisted of individuals with two (homozygous or compound heterozygous) mutations identified, the GJB2(+/-) subgroup consisted of individuals with one heterozygous GJB2 gene mutation identified, and
GJB2-negative subgroup consisted of affected participants with no pathogenic GJB2 gene mutations identified. The GJB2(+) and GJB2(-) subgroups were compared with each other to determine the difference in disease severity, symmetry, and family history.

Data of five individuals of the GJB2(+/-) subgroup with a single autosomal recessive mutation was not included in the genotype - phenotype correlation analysis to avoid bias of ascertainment.

Homogeneity tests were employed to evaluate the impact of the DFNB1 genetic locus on the hearing loss phenotype in the affected group of participants.

Our study results indicate that the severity of hearing loss differs statistically significantly between the GJB2(+) and GJB2(-) subgroups, $p=0.001$ (Table 5, Fig. 2). Profound $\mathrm{HL}$ dominates in the GJB2-positive subgroup

Table 3 Genotype distribution of pathogenic GJB2 gene mutations in the GJB2-positive subgroup of affected participants

\begin{tabular}{|c|c|c|c|c|c|c|}
\hline GJB2 genotype & Profound & Severe & Moderate & Mild & Total count & $\%$ \\
\hline c.[35delG];[35delG] & 30 & 5 & 2 & - & 37 & 44.0 \\
\hline c.[35delG];[313_326del14] & 20 & 4 & 4 & 0 & 28 & 33.3 \\
\hline c.[313_326del14];[313_326del14] & 5 & 1 & 3 & 0 & 9 & 10.7 \\
\hline c.[35delG];[269 T > C] & 1 & - & - & 2 & 3 & 3.6 \\
\hline c.[35delG];[101 T > C] & - & - & - & 2 & 2 & 2.4 \\
\hline c.[35delG];[167delT] & - & - & 1 & - & 1 & 1.2 \\
\hline c.[35delG];[379C > T] & 1 & - & - & - & 1 & 1.2 \\
\hline c.[35delG];[109G > A] & - & - & 1 & - & 1 & 1.2 \\
\hline c.[269 T > C];[313_326del14] & - & - & - & 1 & 1 & 1.2 \\
\hline c.[c.101 T > C];[313_326del14] & - & - & - & 1 & 1 & 1.2 \\
\hline Total GJB2 (+) & 57 & 10 & 11 & 6 & 84 & 100.0 \\
\hline
\end{tabular}


Table 4 Allele frequencies and carrier frequencies of the pathogenic GJB2 gene mutations in the healthy group of Lithuanian population

\begin{tabular}{llll}
\hline Mutation & Count & Allele frequency, \% & Carrier frequency, \% (95 \% Cl) \\
\hline c.101 T> C (p.Met34Thr) rs35887622 & 3 & 1.5 & $3.1(0.6-8.7)$ \\
c.313_326del14 (p.Lys105Glyfs*5) rs111033253 & 2 & 1.0 & $2.0(0.3-7.2)$ \\
c.35delG (p.(Gly12Valfs*2)) rs80338939 & 1 & 0.5 & $1.0(0.1-5.6)$ \\
c.206delT (p.Phe69Serfs*13) & 1 & 0.5 & $1.0(0.1-5.6)$ \\
Total & & & $7.1(2.9-14.2)$ \\
\hline
\end{tabular}

while moderate and mild HL is more common in the GJB2-negative subgroup.

To compare the influence of inactivating (frameshift) and non-inactivating (missense) GJB2 gene mutations on the characteristics of the disease, the GJB2(+) subgroup was divided into two classes of genotypes I/I and I/N (I/ I two inactivating (frameshift) mutations of the GJB2 gene identified; $\mathrm{I} / \mathrm{N}$ inactivating and non-inactivating (missense) mutations of the GJB2 gene in compound heterozygosity identified).

A statistically significant difference in the distribution of HL severity in the classes of the GJB2(+) group was observed, $p=8.005 \times 10^{-11}$ (Table 6, Fig. 3), with profound HL prevailing in the I/I subgroup and mild HL in the $\mathrm{I} / \mathrm{N}$ subgroup.

The influence of GJB2 gene mutations on symmetry of hearing loss was also analysed in affected group of individuals. The difference between GJB2- positive and GJB2-negative subgroups was not statistically significant, $p=0.099$ (Table 7, Fig. 4).

The genealogies of three generation of affected group of individuals were analyzed to assess the heredity of hearing loss. When more than one affected individuals with early onset hearing loss was present in the family assignment to the positive family history was made.

The comparison of GJB2(+) and GJB2(-) subgroups showed a statistically significant difference between the subgroups, $p=0.012$, indicating a more frequent positive family history in the GJB2 (+) subgroup (Table 8, Fig. 5).

We applied logistic regression analysis to evaluate the chances of identifying the two GJB2 gene mutations if profound/severe hearing loss vs moderate/mild hearing loss was diagnosed. OR 3.1 (95 \% CI $1.5-6.6 ; p=0.003$ ) was calculated in our study group of affected individuals

Table 5 Distribution of HL severity of GJB2(-) and GJB2(+) subgroups

\begin{tabular}{llllll}
\hline & Profound HL & Severe HL & Moderate HL & Mild HL & Total \\
\hline GJB2 $(-)$ & 20 & 12 & 19 & 6 & 57 \\
GJB2 $(+)$ & 57 & 10 & 11 & 6 & 84 \\
Total & 77 & 22 & 30 & 12 & 141 \\
Pearson Chi-Square 15.5 & & $p=0.001$ & & \\
Empirical effect size $w=0.7$ & Empirical power $=1.0$ \\
\hline
\end{tabular}

meaning the probability of having GJB2 mutations is 3.1 times higher in case of profound or severe HL (Table 9).

We also estimated the probability of identifying GJB2 gene mutations in affected individuals with a positive family history in our population. The results indicate that the chances are 2.5 times higher than they are in individuals with a negative family history, $p=0.013$ (Table 9).

\section{Discussion}

Hearing loss is considered to be a very heterogeneous disorder. Although many genes have been associated with hearing loss, mutations in the DFNB1 locus are to be the most frequent cause of autosomal recessive hearing loss and routine sequencing of the GJB2 gene and testing of GJB6 gene deletions are recommended in EMQN best practice guidelines [24].

Our study aimed to analyse the impact of DFNB1 locus mutations on the development of early onset hearing loss in an affected group of participants in the Lithuanian population. This group consists of 146 unrelated individuals. Our analysis describes a substantial part: about $0.25-0.5$ per cent of deaf people in Lithuania. The results show a very high proportion of GJB2-positive individuals $(57.5 \%)$ in the research group affected with sensorineural HL compared with other Caucasian populations representing an adequate selection of patients for genetic testing by referring physicians and/or quite high genetic homogeneity in our population. Five individuals (GJB2 (+/-) subgroup), amounting to $3.4 \%$ of unrelated affected participants were found to be heterozygous carriers of one recessive mutation. This result fits into

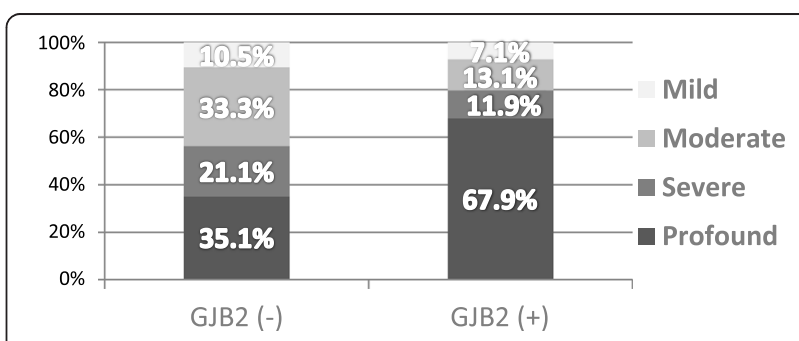

Fig 2 Distribution of HL severity in GJB2(+) and GJB2(-) subgroups of affected group of participants 
Table 6 Distribution of degree of $\mathrm{HL}$ in $\mathrm{I} / \mathrm{I}$ and $\mathrm{I} / \mathrm{N}$ classes of GJB2 (+) subgroup

\begin{tabular}{llllll}
\hline Mutation type & $\begin{array}{l}\text { Profound } \\
\mathrm{HL}\end{array}$ & $\begin{array}{l}\text { Severe } \\
\mathrm{HL}\end{array}$ & $\begin{array}{l}\text { Moderate } \\
\mathrm{HL}\end{array}$ & $\begin{array}{l}\text { Mild } \\
\mathrm{HL}\end{array}$ & Total \\
\hline $\mathrm{I} / \mathrm{I}$ & 55 & 10 & 9 & 0 & 74 \\
$\mathrm{I} / \mathrm{N}$ & 2 & 0 & 2 & 6 & 10 \\
Total & 57 & 10 & 11 & 6 & 84 \\
Pearson Chi-Square 50.0 & & & $p=8.005 \times 10^{-11}$ \\
Empirical effect size $\mathrm{W}=1.6$ & & & Empirical power $=1.0$
\end{tabular}

the $95 \%$ CI of GJB2 gene mutation carrier frequency estimated in our population and possibly represents only carrier status.

Second goal of our study was to assess the burden of DFNB1-related early onset hearing loss in the Lithuanian population. Our group of participants represents the pure ethnic population due to the strict criteria for enrolment guaranteeing the uniqueness of this cohort: all 98 self-reported individuals indicated at least three generations of Lithuanian ethnicity and residency in the same ethno-linguistic region. Although a bigger ethnic population group would better reflect the current state of the amount of carriers of the GJB2 gene mutation, but our results are nevertheless statistically reliable $(\mathrm{p} \leq 0.05)$. The overall frequency of carriers of the GJB2 gene mutation in the healthy group of our study amounted to $7.1 \%$ (approx. - 1 in 14) allowing us to assess the GJB2-associated HL frequency in the Lithuanian population. It was estimated to be approximately 1 in 772 if the assortative marriages didn't distort this value towards the higher edge. The results of the high frequency of carriers of the GJB2 gene mutation in the ethnic Lithuanian groups of healthy participants demonstrate the significant GJB2-associated HL burden in our population.

Though the role of GJB2 and GJB6 gene alterations in the pathogenesis of sensorineural $\mathrm{HL}$ is undisputed, the structure of pathogenic changes identified in different populations is not uniform. The GJB2 gene mutation c.35delG, p.(Gly12Valfs*2) (rs80338939) is the most frequent in Caucasian populations [25] and accounts for up to $70 \%$ of mutated GJB2 gene alleles. The c.167delT,

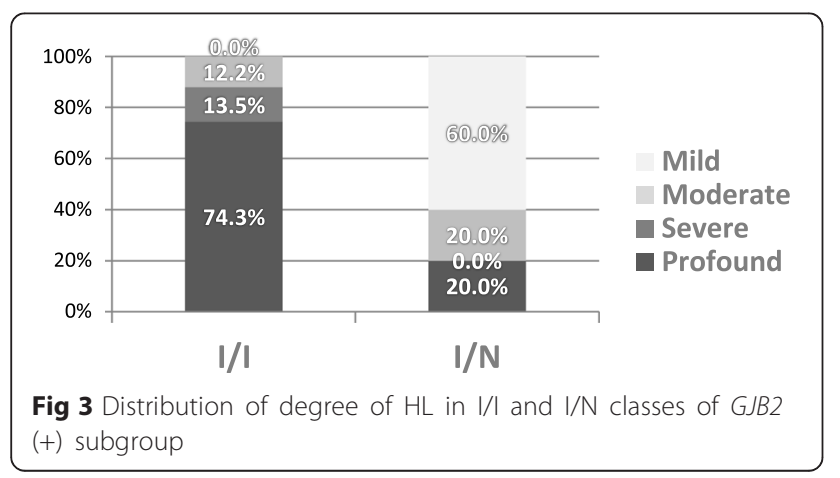

Table 7 Distribution of HL symmetry in GJB2(+) and GJB2(-) subgroups

\begin{tabular}{llll}
\hline & Non symmetric & Symmetric & Total \\
\hline GJB2 $(-)$ & 10 & 47 & 57 \\
GJB2 $(+)$ & 7 & 77 & 84 \\
Total & 17 & 124 & 141 \\
Pearson Chi-Square 2.7 & & $p=0.099$ \\
Empirical effect size $w=0.3$ & & Empirical power $=0.9$ \\
\hline
\end{tabular}

p.(Leu56Argfs*26) (rs80338942) mutation is prevalent in the Ashkenazi Jewish population [26], and c.235delC, p.(Leu79Cysfs*3) (rs80338943) is the leading GJB2 gene mutation in Eastern populations [27]. Splicing mutations (e.g. c. $-23+1 G>A$ (rs80338940)) were found in some populations [28]. The most prevalent GJB2 gene mutation in the affected group of participants of our study was c.35delG, p.(Gly12Valfs*2) (rs80338939). Its allele frequency $(64.7 \%)$ is consistent with many previously published studies in groups of affected individuals of Caucasian populations. The frequency of the c.35delG, p.(Gly12Valfs*2) (rs80338939) mutation in the healthy group in our study $1.0 \%$ is less then described in other Caucasian populations where it can reach $3.2 \%$ [29].

The c.313_326del14, p.(Lys105Glyfs*5) mutation (rs111033253), formerly called c.310del14, c.312del14, and c.314del14, truncates the GJB2 gene and consequently interferes with the structural and functional integrity of connexons. To the best of our knowledge, this mutation has been identified previously in many European populations with a frequency of pathogenic alleles in the affected groups of participants from $0.5 \%$ to $7.3 \%$ (the highest allele frequency $7.3 \%$ occurs in the Polish population (Fig. 6) [30-42]. The high frequency of the c.313_326del14, p.(Lys105Glyfs"5) (rs111033253) (28.32\% of pathogenic alleles) mutation in affected group of participants was an unexpected finding in our study. The c.[313_326del14];[313_326del14] genotype was found in $10.7 \%$ of the GJB2-positive group of unrelated affected participants, suggesting not only a high frequency of carriers of this mutation in our

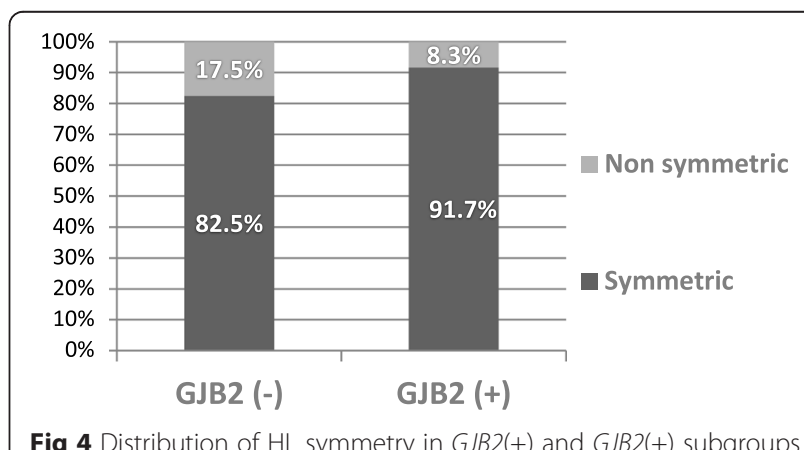

Fig 4 Distribution of HL symmetry in GJB2(+) and GJB2(+) subgroups 
Table 8 Distribution of genealogy types in GJB2(+) and GJB2(-) subgroups

\begin{tabular}{llll}
\hline & Positive family history & Negative family history & Total \\
\hline $\begin{array}{l}\text { GJB2 } \\
(-)\end{array}$ & 17 & 40 & 57 \\
GJB2 & 43 & 41 & 84 \\
$(+)$ & & \\
Total 60 & 81 & 141 \\
Pearson Chi-Square 6.3 & & $p=0.012$ \\
Empirical effect size $w=0.5$ & & Empirical power $=1.0$ \\
\hline
\end{tabular}

population but also its possible origin in Lithuanian ancestors. The high frequency of carriers of the c.313_326del14, p.(Lys105Glyfs*5) (rs111033253) mutation in the entire Lithuanian population is supported by it being identified twice in the ethnic Lithuanian group of healthy participants (a frequency $2.0 \%$ of carriers in the study group). The assumption that there is a high rate of carriers rate of this mutation in the Lithuanian population may also be supported by the coincidental finding of this mutation in the patient with syndromic type of hearing loss - Rogers syndrome [43] and in two affected participants having single GJB2 gene mutation identified and possibly experiencing hearing loss of some other aetiology. The frequency of carriers of this mutation, $4.9 \times 10^{-4}$, has been determined in the NHLBI Exome Sequencing Project in a group of European American descent, showing the extreme rarity of this mutation in the healthy population [44]. The mutation in a homozygous state has been found in 2 out of 12 GJB2-positive study participants (16.7 \%) of Tatar ethnicity in Volga-Ural region of Russia [45]. These numbers are too low to make comprehensive conclusions, but homozygosity itself (with the exception of consanguinity) is a marker of a higher carrier rate in that particular population. In light of the close historical relationship between Lithuanians and Tatars during wars in $8^{\text {th }}-14^{\text {th }}$ century this finding may provide a substantial basis for further analysis or multi-populational research of migration and assimilation processes in Eurasia. Recently

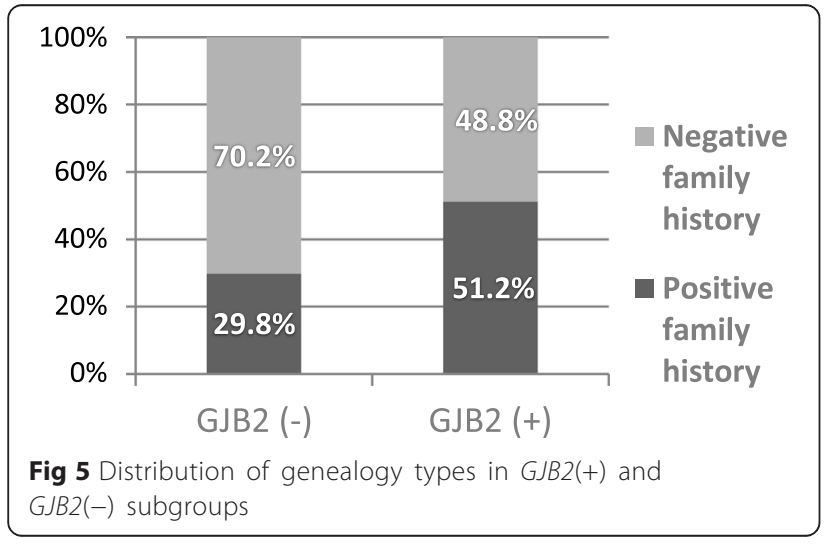

literature review and GJB2 mutations cluster analysis was published where Eastern European descent of the mutation was proposed [46]. Pilot genetic screening of hearing impairment in newborns from Grodno oblast (Belarus) revealed c.313_326del14, p.(Lys105Glyfs*5) (rs111033253) allele frequency $7 \%$ and Polish origin was suggested [47]. From our analysis, we presume its Lithuanian descent. The relatively low frequency of the c.313_326del14, p.(Lys105Glyfs*5) (rs111033253) allele in the Latvian group of affected participants proves that the mutation emerged after the formation of the Baltic tribes. The higher frequencies of the c.313_326del14, p.(Lys105Glyfs*5) (rs111033253) allele amongst neighbouring countries (Poland and Grodno oblast of Belarus) may represent the spreading of the mutation due to close inter-relationships throughout the history of Lithuania.

Besides the undoubtedly disease-causing GJB2 gene mutations, several changes have been disputed in scientific literature regarding their pathogenicity. The frequency of carriers of the c.101 T > C, p.Met34Thr (rs35887622) mutation was determined to be up to $6.5 \%$ in the Caucasian population [29] and initially was reported as a polymorphism. The in silico computational analysis shows contradictory results (Table 2) but later publications explored this variation in functional analysis and concluded it to be pathogenic although with reduced penetrance $[48,49]$. The results of the large UK population study recently published reaffirmed that this variant is associated with mild/moderate HL [50]. The GJB2 mutation c.101 T $>$ C (p.Met34Thr) (rs35887622) was underrepresented in the study group of affected individuals (allelic frequency $2.3 \%$ ) though its carrier rate in the healthy group of Lithuanian population is estimated to be $3.1 \%$. This finding may be explained by lower pathogenicity of the mutation leading to the later and milder manifestation of HL.

Another controversial GJB2 gene change c.109G > A, p.(Val37Ile) (rs72474224) has also been debated. It was previously reported both as polymorphism and a pathogenic mutation [51, 52]. Although bioinformatics tools show some inconsistency in the evaluation of pathogenicity, this mutation is currently classified as pathogenic and associated with a mild to moderate phenotype [53]. The prevalence of c.109G > A, p.(Val37Ile) (rs72474224) was found to be higher in Eastern populations and this mutation was associated with the postnatal development

Table 9 Probabilities of identification of GJB2 gene mutations to affected individual

\begin{tabular}{llll}
\hline $\mathrm{HL}$ characteristics & $\mathrm{OR}(95 \% \mathrm{Cl})$ & $\mathrm{p}$ & Empirical power \\
\hline $\begin{array}{l}\text { Profound/severe vs } \\
\text { moderate/mild HL }\end{array}$ & $3.1(1.5-6.6)$ & 0.003 & 0.9 \\
$\begin{array}{l}\text { Positive family history vs } \\
\text { negative family history }\end{array}$ & $2.5(1.2-5.1)$ & 0.013 & 0.8 \\
\hline
\end{tabular}




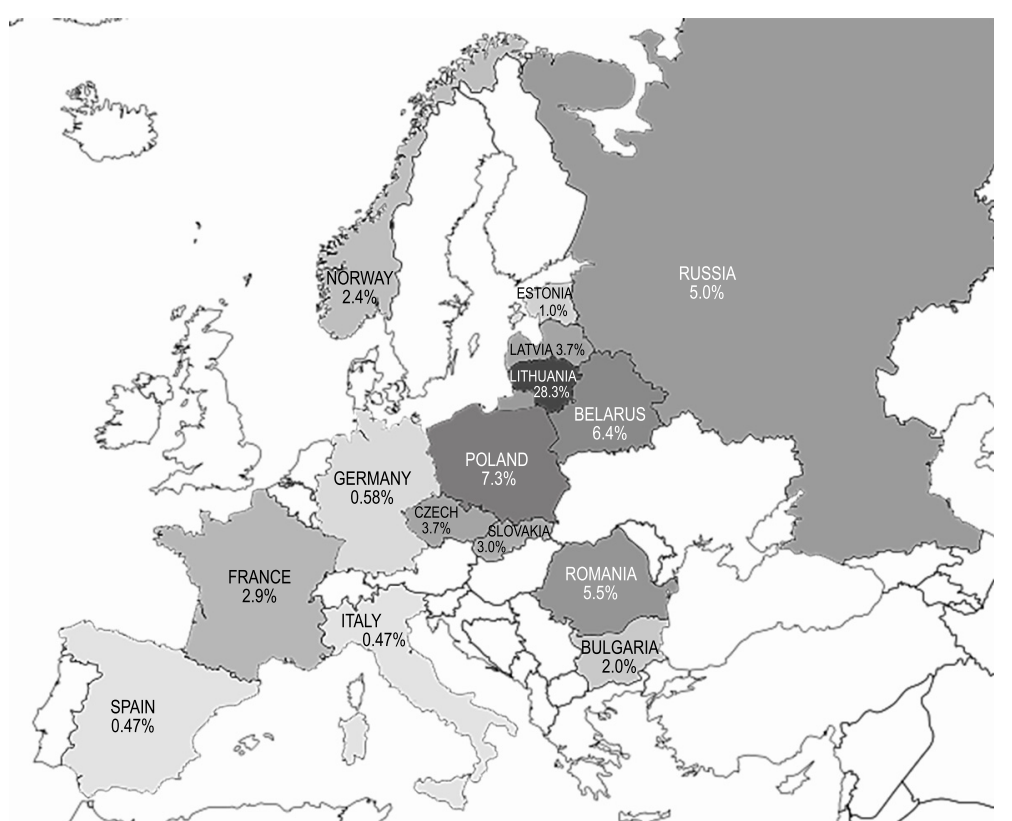

Fig 6 Allele frequencies of c.313_326del14, p.(Lys105Glyfs*5) (rs111033253) mutation in GJB2-positive groups of affected individuals in European populations (see references in Discussion). Adapted from Europe_political_chart available under Creative Commons Attribution-Share Alike 3.0 Unported and GNU Free Documentation Licenses

of HL [54]. In the group of affected individuals we found one nuclear family with the AR mode of sensorineural HL inheritance possessing the aforementioned mutation leading to moderate $\mathrm{HL}$.

The GJB2 gene mutation c.379C $>$ T, p.(Arg127Cys) (rs727503066) is considered to be a variant of uncertain significance in scientific literature [55] and according to in silico analysis (Table 2) but we classified it as a pathogenic mutation. Our decision was based on the observation that the mutation was identified in compound heterozygosity with c.35delG, p.(Gly12Valfs*2) (rs80338939) and with c.101 T > C, p.(Met34Thr) (rs35887622) mutations in two affected family members: father and daughter (the father having genotype c.[101 $\mathrm{T}>\mathrm{C}] ;[379 \mathrm{C}>\mathrm{T}]$ was not enrolled into the study because of the later onset of HL (over 5 years of age). This mutation was also described earlier in scientific literature: it has been identified in a compound heterozygous state with the c.35delG, p.(Gly12Valfs"2) (rs80338939) mutation in an affected individual [22].

GJB6 gene mutations less contribute to the development of hearing loss but several mutations associated with $\mathrm{HL}$ have been described in scientific literature. Gross DFNB1 locus deletions involving the GJB6 gene - $\operatorname{del}(G J B 6$ D13S1830), del(GJB6-D13S1854), $\operatorname{del}(D F N B 1-131 \mathrm{~kb})$ and $\operatorname{del}(D F N B 1>920 \mathrm{~kb})$ which encompass non-translated GJB6 sequences essential for both GJB6 and GJB2 gene transcription have also been implicated in the pathogenesis of sensorineural HL [56]. In Lithuania we have not encountered any affected participant having GJB6 gene point mutations or gross deletions indicating their rarity in our population. These results are similar to the previously published studies in other populations strengthening the evidence that GJB6-related nonsyndromic hearing loss is extremely rare worldwide [57]. In the study group of healthy individuals of Lithuanian origin one carrier of the possibly pathogenic (evidence based on in silico prediction) GJB6 gene mutation was identified.

The results of genotype - phenotype analysis show the significant impact of GJB2 gene mutations on the development of early onset non-syndromic HL in affected group of Lithuanian origin. Our findings indicate that inactivating GJB2 gene mutations were associated with a more severe phenotype than missense mutations - a finding compatible with previous publications [12] and the nature of the mutations. GJB2 mutations also in general lead to more severe HL (OR 3.1, $p=0.003$ ) with positive family history (OR 2.5, $p=0.013$ ), compared with the non-GJB2 aetiology of HL in Lithuanian population. Several studies have made comparisons of the characteristics of HL between GJB2-related and GJB2negative groups of affected individuals, and statistically significant differences were determined in genealogy but not in disease severity or other HL characteristics [58]. These observations may be helpful in clinical settings to prognosticate the results of genetic testing and disease course to the patients with HL in the Lithuanian population. 


\section{Conclusions}

Analysis of the frequency of the pathogenic GJB2 gene allele in the Lithuanian population revealed a high proportion of c. 313_326del14 mutations in the GJB2-positive group suggesting its possible origin in the ancestors of the Lithuanian population. The findings of high frequency of carriers of the GJB2 gene mutation in the Lithuanian group of healthy participants led to the estimation of significant ( 1 in 772) GJB2-associated HL frequency in Lithuania. The findings of the study quantified the impact of mutations in the DFNB1 genetic region to the development of $\mathrm{HL}$ in the Lithuanian population indicating the significant contribution of the GJB2 gene mutations on the pathogenesis of the disorder. The results are useful for establishing the principles to predict the course of the disease in the patients with early onset of hearing loss.

\section{Availability of supporting data}

The data sets supporting the results of this article are included within the article and its additional files.

\section{Additional files}

\section{Additional file 1: The conditions for PCR amplification of GJB2 and} GJB6 genes coding sequences (primer sequences, annealing temperature, lengths of the amplicons). (PDF $8 \mathrm{~kb}$ )

Additional file 2: Dataset of the group of affected individuals (DEAFGEN project). (PDF $191 \mathrm{~kb}$ )

Additional file 3: Dataset of the group of ethnic Lithuanian population (LITGEN project). (PDF $138 \mathrm{~kb}$ )

\section{Abbreviations}

CHL: congenital hearing loss; DEAFGEN: the abbreviation of the project The genomics of congenital/hereditary hearing loss: implication in disease pathogenesis, influence to phenotypic expression and treatment efficiency;; DFNB1 locus: deafness, autosomal recessive 1 locus; EMQN: european molecular genetics quality network; GJB2 and GJB6 genes: gap junction protein, beta-2 and beta- 6 genes; GJB2(+): GJB2-positive group of affected individuals (2 pathogenic GJB2 gene mutations identified); GJB2(+/-): 1 pathogenic GJB2 gene mutation identified; GJB2(+): GJB2-negative group of affected individuals (no pathogenic GJB2 gene mutation identified); HL: hearing loss; I/I: subgroup of $G J B 2(+)$ group with 2 inactivating mutations identified; I/N: subgroup of GJB2(+) group with 1 inactivating and 1 non-inactivating mutations identified; LITGEN: the abbreviation of the project the 'Genetic diversity of the population of Lithuania and changes of its genetic structure related with evolution and common diseases'; OMIM: online mendelian inheritance in man; OR: odds ratio.

\section{Competing interests}

The authors declare that they have no competing interests.

\begin{abstract}
Authors' contributions
Conception and design of study: $V M, V K$ and $A U$. Acquisition of data: $V M, A U$, $E P, B B, B T, A M, L C, L A, I U, I D, T R, J B, E G$, and EL. Analysis and/or interpretation of data: VM, JB, TR, AJ. Drafting the manuscript: VM, JB, IU, and AJ. Revision of the manuscript: $E P, B B, B T, A M, L C, L A, I U, I D, T R, J B, E G, E L, A J, V K$, and $A U$. All authors approved the final manuscript.
\end{abstract}

\section{Acknowledgements}

We thank the patients and their parents for permitting to present the clinical and laboratory data together with the results of the genetic analysis.
The research leading to these results is part of the LITGEN project (VP1-3.1-ŠMM-07-K-01-013) and was funded by the European Social Fund under the Global Grant measure.

\section{Author details}

${ }^{1}$ Department of Human and Medical Genetics, Faculty of Medicine, Vilnius University, Vilnius, Lithuania. ${ }^{2}$ Centre of Ear, Nose and Throat Diseases, Vilnius University Hospital Santariskiu clinics, Vilnius, Lithuania.

Received: 19 October 2015 Accepted: 15 February 2016

Published online: 19 February 2016

\section{References}

1. Morton CC, Nance WE. Newborn hearing screening - a silent revolution. N Engl J Med. 2006:354(20):2151-64.

2. Fortnum HM, Davis A, Summerfield AQ, Marshall DH, Davis AC, Bamford JM et al. Prevalence of permanent childhood hearing impairment in the United Kingdom and implications for universal neonatal hearing screening: questionnaire based ascertainment study. BMJ. 2001;323(7312):536-40.

3. De Keulenaer S, Hellemans J, Lefever S, Renard J-P, De Schrijver J, Van de Voorde $\mathrm{H}$, et al. Molecular diagnostics for congenital hearing loss including 15 deafness genes using a next generation sequencing platform. BMC Med Genomics. 2012;5:17

4. Smith RJH, Shearer AE, Hildebrand MS, Van Camp G. Deafness and Hereditary Hearing Loss Overview. GeneReviews ${ }^{\oplus}$. 1993-2015. http://www.ncbi.nlm.nih.gov/books/NBK1434/.

5. Toriello HV, Smith SD. Hereditary Hearing Loss and Its Syndromes. New York: Oxford University Press; 2013. 732 p.

6. Hereditary Hearing Loss Homepage [Internet]. [cited 29 July, 2015]. Available from: http://hereditaryhearingloss.org.

7. Putcha GV, Bejjani BA, Bleoo S, Booker JK, Carey JC, Carson N, et al. A multicenter study of the frequency and distribution of GJB2 and GJB6 mutations in a large North American cohort. Genet Med. 2007;9(7):413-26.

8. Tang H-Y, Fang P, Ward PA, Schmitt E, Darilek S, Manolidis S, et al. DNA Sequence analysis of GJB2, encoding connexin 26: observations from a population of hearing impaired cases and variable carrier rates, complex genotypes, and ethnic stratification of alleles among controls. Am J Med Genet Part A. 2006;140(22):2401-15.

9. Stenson PD, Mort M, Ball EV, Shaw K, Phillips AD, Cooper DN. The human gene mutation database: building a comprehensive mutation repository for clinical and molecular genetics, diagnostic testing and personalized genomic medicine. Hum Genet. 2014;133(1):1-9

10. Oyamada M, Takebe K, Oyamada Y. Regulation of connexin expression by transcription factors and epigenetic mechanisms. Biochim et Biophys Acta. 2013;1828(1):118-33.

11. Bargiello TA, Tang Q, Oh S, Kwon T. Voltage-dependent conformational changes in connexin channels. Biochim et Biophys Acta. 2012;1818(8):1807-22.

12. Snoeckx RL, Huygen PLM, Feldmann D, Marlin S, Denoyelle F, Waligora J, et al. GJB2 mutations and degree of hearing loss: a multicenter study. Am J Hum Genet. 2005;77(6):945-57.

13. Lee JR, White TW. Connexin-26 mutations in deafness and skin disease. Expert Rev Mol Med. 2009:11, e35.

14. Ye J, Coulouris G, Zaretskaya I, Cutcutache I, Rozen S, Madden TL. PrimerBLAST: a tool to design target-specific primers for polymerase chain reaction. BMC Bioinformatics. 2012;13:134

15. Cooper DN, Krawczak M. Human Gene Mutation. BIOS Scientific Publishers; 1993.

16. Connexins and deafness Homepage. [Internet]. Available from: http://davinci.crg.es/deafness/.

17. Del Castillo FJ, Rodriguez-Ballest M, Alvarez A, Hutchin T, Leonardi E, de Oliveira CA, et al. A novel deletion involving the connexin-30 gene, del(GJB6-d13s1854), found in trans with mutations in the GJB2 gene (connexin-26) in subjects with DFNB1 non-syndromic hearing impairment. J Med Genet. 2005;42(7):588-94.

18. McKenna A, Hanna M, Banks E, Sivachenko A, Cibulskis K, Kernytsky A, et al. The genome analysis toolkit: a mapreduce framework for analyzing next-generation DNA sequencing data. Genome Res. 2010;20(9):1297-303.

19. DePristo MA, Banks E, Poplin RE, Garimella KV, Maguire JR, Hartl C, et al. A framework for variation discovery and genotyping using next-generation DNA sequencing data. Nat Genet. 2011;43(5):491-8. 
20. Wang K, Li M, Hakonarson H. ANNOVAR: functional annotation of genetic variants from high-throughput sequencing data. Nucleic Acids Res. 2010;38(16):e164.

21. Robinson JT, Thorvaldsdóttir H, Winckler W, Guttman M, Lander ES, Getz G, et al. Integrative genomics viewer. Nat Biotechnol. 2011;29(1):24-6.

22. Dahl HHM, Saunders K, Kelly TM, Osborn AH, Wilcox S, Cone-Wesson B, et al. Prevalence and nature of connexin 26 mutations in children with non-syndromic deafness. Med J. 2001;175(4):191-4.

23. Adzhubei IA, Adzhubei S, Peshkin L, Ramensky VE, Gerasimova A, Bork P et al. A method and server for predicting damaging missense mutations. Nat Methods. 2010;7:248-9.

24. Hoefsloot LH, Roux A-F, Bitner-Glindzicz M. EMQN best practice guidelines for diagnostic testing of mutations causing non-syndromic hearing impairment at the DFNB1 locus. Eur J Hum Genet. 2013;21(11):1325-9.

25. Estivill X, Fortina P, Surrey S, Rabionet R, Melchionda S, D'Agruma L, et al. Connexin-26 mutations in sporadic and inherited sensorineural deafness. Lancet. 1998:351(9100):394-8.

26. Morell RJ, Kim HJ, Hood L, Goforth L, Friderici K, Fisher R, et al. Mutations in the connexin 26 gene (GJB2) among Ashkenazi Jews with nonsyndromic recessive deafness. N Engl J Med. 1998;339(21):1500-5.

27. Ohtsuka A, Yuge I, Kimura S, Namba A, Abe S, Van Laer L, et al. GJB2 deafness gene shows a specific spectrum of mutations in Japan, including a frequent founder mutation. Hum Genet. 2003;112(4):329-33.

28. Tekin M, Xia X-J, Erdenetungalag R, Cengiz FB, White TW, Radnaabazar J, et al. GJB2 mutations in Mongolia: complex alleles, low frequency, and reduced fitness of the deaf. Ann Hum Genet. 2010;74(2):155-64.

29. Database of Single Nucleotide Polymorphisms (dbSNP). [Internet]. Available from: http://www.ncbi.nlm.nih.gov/SNP/.

30. Rabionet R, Zelante L, López-Bigas N, D’Agruma L, Melchionda S, Restagno $\mathrm{G}$, et al. Molecular basis of childhood deafness resulting from mutations in the GJB2 (connexin 26) gene. Hum Genet. 2000;106(1):40-4.

31. Kupka S, Braun S, Aberle S, Haack B, Ebauer M, Zeißler U, et al. Frequencies of GJB2 mutations in German control individuals and patients showing sporadic non-syndromic hearing impairment. Hum Mutat. 2002;20(1):77-8.

32. Marlin S, Feldmann D, Blons H, Loundon N, Rouillon I, Albert S. GJB2 and GJB6 mutations: genotypic and phenotypic correlations in a large cohort of hearing-impaired patients. Arch of Otolaryngol Head Neck Surg. 2005;131(6):481-7.

33. Seeman $P$, Malíková M, Rašková $D$, Bendová $O$, Groh $D$, Kubálková $M$, et al. Spectrum and frequencies of mutations in the GJB2 (Cx26) gene among 156 Czech patients with pre-lingual deafness. Clin Genet. 2004;66(2):152-7.

34. Wiszniewski W, Sobieszczanska-Radoszewska L, Nowakowska-Szyrwinska E, Obersztyn E, Bal J. High frequency of GJB2 gene mutations in polish patients with prelingual nonsyndromic deafness. Genet Test. 2001;5(2):147-8.

35. Sterna O, Pronina N, Grinfelde I, Kuske S, Krumina A, Lugovska R, et al. Spectrum and frequency of the GJB2 gene mutations among Latvian patients with prelingual nonsyndromic hearing loss. Proc Latvian Acad Sci, Section B. 2009;63(4/5):198-203.

36. Danilenko N, Merkulava E, Siniauskaya M, Olejnik O, Levaya-Smaliak A, Kushniarevich A, et al. Spectrum of genetic changes in patients with non-syndromic hearing impairment and extremely high carrier frequency of 35delG GJB2 mutation in Belarus. PLoS One. 2012;7(5), e36354.

37. Minárik G, Tretinárová D, Szemes T, Kádasi L’. Prevalence of DFNB1 mutations in Slovak patients with non-syndromic hearing loss. I Int J Pediatr Otorhinolaryngol. 2012:76(3):400-3.

38. Rădulescu L, Mârțu C, Birkenhäger R, Cozma S, Ungureanu L, Laszig R. Prevalence of mutations located at the DFNB1 locus in a population of cochlear implanted children in eastern Romania. Int J Pediatr Otorhinolaryngol. 2012;76(1):90-4.

39. Teek R, Kruustük K, Zordania R, Joost K, Reimand T, Möls T, et al. Prevalence of c.35delG and p.M34T mutations in the GJB2 gene in Estonia. Int J Pediatr Otorhinolaryngol. 2010;74(9):1007-12.

40. Bliznetz E, Galkina V, Matyushchenko G, Kisina A, Markova T, Polyakov A. Changes in the connexin 26 (GJB2) gene in Russian patients with hearing disorders: results of long-term molecular diagnostics of hereditary nonsyndromic deafness. Genetika. 2012:48(1):112-24.

41. Siem G, Fagerheim T, Jonsrud C, Laurent C, Teig E, Harris S, et al. Causes of hearing impairment in the Norwegian paediatric cochlear implant program. Int J Audiol. 2010;49(8):596-605.

42. Popova D, Kaneva R, Varbanova S, Popov T. Prevalence of GBJ2 mutations in patients with severe to profound congenital nonsyndromic sensorineural hearing loss in Bulgarian population. Eur Arch OtoRhinoLaryngol. 2012:269(6):1589-92.

43. Mikstiene V, Songailiene J, Byckova J, Rutkauskiene G, Jasinskiene E, Verkauskiene $\mathrm{R}$, et al. Thiamine responsive megaloblastic anemia syndrome: a novel homozygous SLC19A2 gene mutation identified. Am J Med Genet A. 2015;167(7):1605-9.

44. Exome Variant Server, NHLBI GO Exome Sequencing Project (ESP) [Internet]. [cited 03 June, 2015]. Available from: http://evs.gs.washington.edu/EVS/.

45. Dzhemileva L, Khidiyatova I, Khusnutdinova E. Molecular screening of deafness in populations and patients with nonsyndromic congenital deafness from the Volga-Ural region of Russia. In: Krause DFaR, editor. Deafness, Hearing Loss and the Auditory System: Nova Science Publishers, Inc., New York, USA; 2009.

46. Tsukada K, Nishio S-y, Hattori M, Usami S-I. Ethnic specific spectrum of GJB2 and SLC26A4 mutations: their origin and a literature review. Ann Otol Rhinol Laryngol. 2015;124(1 suppl):61S-76.

47. Bliznets EA, Marcul' DN, Khorov OG, Markova TG, Poliakov AV. The mutation spectrum of the GJB2 gene in Belarussian patients with hearing loss. Results of pilot genetic screening of hearing impairment in newborns. Genetika. 2014;50(2):214-21.

48. Griffith AJ, Friedman TB. Auditory function and the M34T allele of connexin 26. Archives of Otolaryngol Head Neck Surg. 2002;128(1):94.

49. Bicego M, Beltramello M, Melchionda S, Carella M, Piazza V, Zelante L, et al. Pathogenetic role of the deafness-related M34T mutation of Cx26. Hum Mol Genet. 2006;15(17):2569-87.

50. Hall A, Pembrey M, Lutman M, Steer C, Bitner-Glindzicz M. Prevalence and audiological features in carriers of GJB2 mutations, C.35delG and C.101 T > C (p.M34T), in a UK population study. BMJ Open. 2012;2(4):e001238.

51. Kelley PM, Harris DJ, Comer BC, Askew JW, Fowler T, Smith SD, et al. Novel mutations in the connexin 26 gene (GJB2) that cause autosomal recessive (DFNB1) hearing loss. Am J Hum Genet. 1998;62(4):792-9.

52. Roux A-F, Pallares-Ruiz N, Vielle A, Faugère $V$, Templin C, Leprevost $D$, et al. Molecular epidemiology of DFNB1 deafness in France. BMC Med Genet. 2004;5:5.

53. Wattanasirichaigoon D, Limwongse $C$, Jarieng prasert C, Yenchitsomanus PT, Tocharoenthanaphol C, Thongnoppakhun W, et al. High prevalence of V37I genetic variant in the connexin-26 (GJB2) gene among non-syndromic hearing-impaired and control Thai individuals. Clin Genet. 2004:66(5):452-60.

54. Li L, Lu J, Tao Z, Huang Q, Chai Y, Li X, et al. The p.V37I Exclusive genotype of GJB2: a genetic risk indicator of postnatal permanent childhood hearing impairment. PLoS One. 2012;7(5), e36621.

55. Landrum MJ, Lee JM, Riley GR, Jang W, Rubinstein WS, Church DM, et al ClinVar: public archive of relationships among sequence variation and human phenotype. Nucleic Acids Res. 2014;42(Database issue):D980-5.

56. Del Castillo I, Moreno-Pelayo MA, del Castillo FJ, Brownstein Z, Marlin S, Adina Q, et al. Prevalence and evolutionary origins of the del(GJB6D13S1830) mutation in the DFNB1 locus in hearing-impaired subjects: a multicenter study. Am J Hum Genet. 2003;73(6):1452-8.

57. Amorini M, Romeo P, Bruno R, Galletti F, Di Bella C, Longo P, et al. Prevalence of deafness-associated connexin-26 (GJB2) and connexin-30 (GJB6) pathogenic alleles in a large patient cohort from eastern Sicily. Ann Hum Genet. 2015;79(5):341-9.

58. Schimmenti LA, Martinez A, Telatar M, Lai C-H, Shapiro N, Fox M, et al. Infant hearing loss and connexin testing in a diverse population. Genet Med. 2008;10(7):517-24.

\section{Submit your next manuscript to BioMed Central and we will help you at every step:}

- We accept pre-submission inquiries

- Our selector tool helps you to find the most relevant journal

- We provide round the clock customer support

- Convenient online submission

- Thorough peer review

- Inclusion in PubMed and all major indexing services

- Maximum visibility for your research

Submit your manuscript at www.biomedcentral.com/submit 